\title{
Remerciements à nos évaluateurs de 2017
}

Nous tenons à remercier les personnes suivantes pour leur contribution inestimable en tant qu'évaluateurs pour la revue Promotion de la santé et prévention des maladies chroniques au Canada en 2017. Leur expertise contribue grandement à la qualité de notre revue et à la diffusion des nouvelles connaissances auprès de la communauté scientifique, au Canada comme à l'échelle internationale.

Amanuel Abajobir

Alexander Allen

Laura Arbour

Christopher Ardern

Chris Barrington-Leigh

Marco Battaglia

William Bellew

Nicholas Birkett

Tara Boelsen-Robinson

Lisa Bourque Bearskin

Randy Boyes

Mayilee Canizares

Nick Cavill

Bernard Choi

Brydie Clarke

Adam Cole

Yang Cui

Jon Doan

Rachel Engler-Stringer

Gagan Fervaha

Shawn Fraser

Daniel Fuller

Didier Garriguet

Gary Goldfield

Samantha Gontijo-Guerra

Samantha Goodman

Linda Greene-Finestone

Grant Handrigan

Anthony Hanley
Rhona Hanning
Erin Hoare
Erin Hobin
Sheilah Hogg-Johnson
Wendy Hovdestad
Ilene Hyman
Brian Hyndman
Amanda Jones
Glenn Keays
Ryan David Kennedy
Alexandra Kent
Sharon Kirkpatrick
Primoz Kocbek
Vicki Kristman
Eric Lavigne
Justine Leavy
Yann Le Bodo
Eun-Young Lee
Isra Levy
Shiliang Liu
Emily Lowthian
Peter Macaulay
Zack Marshall
Lindsay McLaren
Amy Metcalfe
Stephanie Montesanti
Howard Morrison
Sil

Yeeli Mui

Patti-Jean Naylor

Cory Neudorf

Alvaro Osornio-Vargas

Marie-Claude Paquette

Alex Price

Stephanie Prince Ware

Kim Raine

Liam Remillard

Kim Robien

Kaley Roosen

Laura Rosella

Sarah Rotz

Richard Sadler

Travis Saunders

Mary Scourboutakos

Gabriel Shapiro

Shahin Shooshtari

Kelly Skinner

Anna Syrowatka

Valerie Tarasuk

Joan Tranmer

Cynthia Weijs

Ellen M. Wells

Russell Wilkins

Jinhui Zhao 